\title{
Plus belle la vie : analyse des interactions et sélection d'un corpus pour l'apprentissage du français langue étrangère
}

\author{
Virginie André ${ }^{*}$ \\ ${ }^{1}$ ATILF, Université de Lorraine et CNRS
}

\begin{abstract}
Résumé. Afin de pallier le manque de ressources multimédia présentant des situations de communications authentiques de la vie quotidienne, nous avons choisi, analysé et utilisé des séquences de la série Plus belle la vie à des fins d'apprentissage du Français Langue Etrangère (FLE). Dans cet article, nous exposons tout d'abord les arguments en la faveur de l'utilisation de cette série. Ensuite, nous présentons les analyses langagières et interactionnelles des dialogues afin d'examiner s'ils sont proches ou éloignés d'interactions verbales authentiques. Un corpus est ensuite constitué à partir notamment d'une sélection des dialogues jugés proches du français parlé en interaction. Enfin, nous rendrons compte brièvement des premières expérimentations de l'utilisation de ce corpus à des fins d'apprentissage du français. Nous verrons que l'approche que nous mettons en place s'inscrit dans le cadre de l'utilisation des corpus multimédia à des fins didactiques, bien que le corpus Plus belle la vie soit particulier.
\end{abstract}

\footnotetext{
* Virginie.Andre@univ-lorraine.fr
} 


\begin{abstract}
Plus belle la vie : analysis of interactions and selection of a corpus for learning French as a foreign

language. In order to make up for the lack of multimedia resources presenting authentic everyday communication situations, we have chosen, analyzed and used sequences from the series Plus belle la vie for the purpose of learning French as a Foreign Language (FLE). In this paper, we begin with the arguments in favour of using this series. Next, we present the language and interactive analyses of dialogues in order to examine whether they are close or distant from authentic verbal interactions. A corpus is then formed from a selection of dialogues judged to be close to French spoken in interaction. Finally, we will give a brief account of the first experiments on the use of this corpus for learning French. We will see that the approach we are using is part of the use of multimedia corpora for didactic purposes, although the Plus belle la vie corpus is special.
\end{abstract}

\title{
Introduction
}

Les corpus multimédia de situations de communication authentiques que les enseignants et les apprenants de Français Langue Etrangère (FLE) peuvent utiliser à des fins d'apprentissage sont encore rares. Depuis que les travaux en didactique des langues ont mis au jour l'importance de la confrontation des apprenants à la langue cible, différents documents supports ont été utilisés. Parmi les plus fréquents, nous trouvons les films, les émissions de télévisions ou les documentaires. Leur fréquence est liée à leur accessibilité. Cependant, ces documents, et notamment les films, donnent à entendre aux apprenants des interactions scriptées, rédigées à l'avance par un scénariste ou un dialoguiste. Leur degré d'authenticité est souvent moindre et finalement l'utilisation des films n'est pas très éloignée de celle des dialogues construits des manuels. Nous nous sommes donc interrogée sur les différentes façons de pallier ce manque de documents authentiques. Force est de constater que la création de corpus multimédia est longue et couteuse, nous nous sommes orientée vers les séries télévisées en étudiant la possibilité d'une exploitation didactique.

Depuis plusieurs années, la télévision propose des séries de courte durée cherchant à mettre en scène le quotidien des français. Parmi ces séries, nous 
avons choisi Plus belle la vie parce qu'elle existe depuis 2004, qu'elle a un taux d'audience important (autour de $20 \%$ ) et surtout parce qu'elle prétend justement montrer les préoccupations quotidiennes des Français, et plus précisément des habitants d'un quartier de Marseille. Dans un premier temps, nous avons analysé les interactions de différents épisodes à la lumière de ce que nous savons des interactions verbales réelles, authentiques non scriptées. Nous avons défini plusieurs éléments et critères d'analyse qui nous ont permis de comparer les interactions, scriptées et non scriptées. Ces premières analyses montrent que certaines séquences, c'est-à-dire certains passages d'un épisode, ne diffèrent pas de manière significative des interactions authentiques. Dans un deuxième temps, nous avons écarté les séquences interactionnelles scriptées trop éloignées des interactions réelles afin de créer un corpus utilisable à des fins d'apprentissage du français. Dans un troisième temps, nous avons testé ce corpus avec des apprenants et des enseignants de FLE.

\section{De la rareté des corpus multimédia authentiques à Plus belle la vie}

L'idée d'utiliser une série télévisée à des fins d'apprentissage du français peut paraître fortement incohérente pour quelqu'un qui préconise l'utilisation de documents authentiques présentant de réelles situations de communication. Cependant, beaucoup d'enseignants de FLE, quelle que soit la structure à laquelle ils appartiennent, se plaignent de ne pas pouvoir montrer à leurs apprenants des vidéos de la vie quotidienne des Français. Malgré la prolifération des films en tout genre sur Internet, les interactions authentiques restent rares. En outre, même si le nombre et la taille des corpus oraux et multimédia constitués et exploités par les linguistes sont actuellement en net accroissement, leur accessibilité reste peu aisée pour des enseignants de FLE. En effet, nous pouvons citer les projets CLAPI-FLE (http://clapi.ish-lyon.cnrs.fr/FLE/) et FLEURON (https://apps.atilf.fr/fleuron/) qui réfléchissent à l'utilisation de corpus multimédia dans des dispositifs d'apprentissage du français. Néanmoins, les interactions authentiques filmées et accessibles à des enseignants restent insuffisantes pour proposer à des apprenants du français une exposition variée à la langue.

Cette plainte des enseignants de FLE nous a conduit à tenter de trouver une solution, une sorte de compromis entre des situations de communications 
authentiques filmées et des films de situations de communication jouées par des acteurs. Il n'est pas rare de constater en cours de FLE que le document authentique permettant aux apprenants d'être exposé à la langue cible soit le film cinématographique. Or, dans la quasi-totalité des films, les dialogues sont très scriptés et sont rédigés avec une attention particulière portée à l'esthétique plutôt qu'à la réalité. Nous avons tout d'abord fait l'hypothèse que cela pouvait être différent avec des séries télévisées, au moins avec un certain type de séries. Les séries télévisées françaises étant en plein essor, nous avons donc décidé de nous y intéresser. Leur format, de courte durée, a également attiré notre attention. Ensuite, certaines séries télévisées françaises créent leurs intrigues à partir de la vie quotidienne. Après quelques recherches dans un monde totalement inconnu pour nous, nous avons pensé à des séries françaises du type Un gars une fille qui met en scène un couple dans son quotidien ou Parents mode d'emploi qui présente la vie d'une famille sur un ton humoristique et à Plus belle la vie. En réalisant une petite enquête auprès de personnes connaissant ces séries ainsi qu'auprès des étudiants du Master Sciences du langage et didactique des langues de l'Université de Lorraine à Nancy à qui nous avions soumis notre problématique, nous avons choisi Plus belle la vie. Nous reviendrons sur les arguments qui ont plaidé en la faveur de cette série. Une fois ce choix effectué, la principale question qui a motivé cette étude a été : Plus belle la vie ou rien? En d'autres termes, nous nous sommes interrogée sur la pertinence de l'utilisation d'un tel support en formation linguistique. Nous nous sommes demandée s'il était envisageable d'utiliser la série en cours de FLE ou s'il fallait absolument éviter. Nous avons étudié les objectifs et les thématiques abordées dans la série puis analysé les interactions produites par les acteurs. Nous nous sommes également inspirée de travaux similaires portant sur des séries ou des films américains $[1,2]$. Nous présenterons cette analyse dans le point suivant.

Le choix de cette série est tout d'abord lié à ses objectifs et aux thématiques abordées. Plus belle la vie, selon plusieurs sites Internet qui lui sont dédiés, met en scène le quotidien des habitants d'un quartier de Marseille. Depuis 2004, la série est diffusée sur France 3, du lundi au vendredi vers $20 \mathrm{~h} 20$ et remporte un grand succès auprès de plus de cinq millions de téléspectateurs. Cette série est présentée par ses producteurs comme une série réaliste. Ce caractère réaliste ou vraisemblable a même été scientifiquement discuté [3,4]. L'objectif premier de la série est de diffuser des scènes de vie quotidienne dans lesquelles chaque spectateur peut se retrouver. Même si la série propose de plus en plus, au cours de ces dernières années, des intrigues policières ou fantastiques [5], les scènes de la vie quotidienne demeurent une des priorités de la série. Dans tous les épisodes, 
nous pouvons par exemple assister à des discussions dans un bar, des échanges entre adolescents, des explications de problèmes financiers, des visites d'appartement, des scènes de cuisine, des conversations à bâtons rompus, etc.

En outre, diffusée sur une chaine du service public, la série se donne également des objectifs sociaux. A propos de la dimension politique de la série, Byron-Portet [6, p. 101] explique que "Plus belle la vie utilise le quartier comme une loupe grossissante afin d'explorer des thèmes capables de concerner l'ensemble des Français ». La série aborde régulièrement des problématiques sociales, nationales ou internationales telles que : l'homosexualité, le chômage, le viol, l'avortement, la prostitution, le SIDA, le cancer, la drogue, l'alcoolisme, la mafia, l'écologie, la sécurité, l'immigration, l'accueil des réfugiés ou la mondialisation. Chacune de ces thématiques, lorsqu'elle est abordée dans la série, fait l'objet de discussions et d'échanges d'arguments entre les personnages. Ces interactions ont lieu sur, ou autour de, la place centrale du quartier imaginaire de la série : «Espace de convivialité, voire de sociabilité, la place du Mistral est aussi un espace public où des individus débattent de sujets d'actualité, relatifs à des événements ponctuels et éphémères, et de problèmes de société davantage intemporels » (Ibid.). Un autre avantage de cette série, dans le cadre de notre étude, est que les épisodes sont tournés peu de temps avant leur diffusion (généralement trois à cinq semaines avant). Par conséquent, les thèmes abordés sont d'actualité, ils correspondent à des grands événements nationaux et internationaux (comme la fête de la musique, les examens à l'université, les fêtes de fin d'année, la journée des droits de la femme, etc.) ou à des événements ponctuels importants et sensibles (comme les attentats, l'identité nationale, le mariage gay). Dans les études menées sur la série, certains voient des liens entre le traitement de la délinquance, la mise en valeur des policiers et du commissariat, et le cheval de bataille - l'insécurité - de Nicolas Sarkozy pendant sa campagne présidentielle [6]. D'autres remarquent dans la presse, que la série met en scène un personnage qui va cacher de l'argent en Suisse en même temps que Jérôme Cahuzac [7]. Quel que soit l'événement traité et la façon de le traiter, les producteurs tentent d'être au plus près du réel et parfois de l'actualité. La production explique que la série défend des valeurs de respect, de tolérance, d'humanisme et de citoyenneté. En résumé, la variété des thèmes abordés semble être susceptible d'offrir une grande variété de ressources pour l'enseignement et l'apprentissage du français.

Enfin, au-delà des thèmes abordés par la série, le souci du réalisme et de l'adéquation à la réalité est constant chez les scénaristes de la série [5]. Cette précaution est importante dans le cadre de notre étude et semble être un 
argument en faveur de l'utilisation de cette série à des fins didactiques. Il semble que le réalisme soit recherché à différents niveaux, par exemple dans les réactions des personnages ou encore dans le lexique des dialogues. Ainsi, si l'intrigue peut parfois être invraisemblable, notamment lors d'intrigues policières ou fantastiques, «le principal est que les réactions soient justes » (Ibid. : p. 63). De plus, une attention particulière est portée sur l'adéquation des termes utilisés avec la réalité. En analysant une "péripétie tout au long $\mathrm{du}$ processus de production ", Mille [5] rend compte des étapes qui permettent de rendre l'événement crédible. Parmi ces étapes, l'auteure explique que les scénaristes portent une attention à la justesse de leur propos par rapport à la réalité. Par exemple, un délit est commis par un adolescent, la question des années de prison est posée et des renseignements sur les aspects juridiques et légaux sont collectés. Par ailleurs, lorsqu'un personnage est hospitalisé, une enquête est réalisée afin d'équiper le décor avec les bons appareils et d'utiliser le lexique médical approprié. «Ce qui est en jeu ici, c'est bien de faire correspondre le sens de ce qui se passerait probablement dans la vie avec ce qui se produit dans la série » (Ibid. : p. 62). Ainsi, Mille [5] explique que les termes prononcés par les acteurs sont choisis avec soin, que « le choix des termes justes fonctionne comme une précision de l'effet de réel visé » et que « les termes cités fonctionnent comme des points d'ancrage dans une réalité » (Ibid. : p. 65). Ce que nous pouvons remarquer, c'est qu'en ce qui concerne la réalité des dialogues, seul le lexique semble être important. D'un point de vue du contenu des dialogues, la pertinence des arguments est également une préoccupation importante ainsi que l'intelligibilité pour les téléspectateurs. Cependant, nous ne savons rien (et nos questions posées aux producteurs de la série sont restées sans réponse) sur la façon dont les interactions sont rédigées. Est-ce qu'elles sont élaborées également dans un souci du réel ? Est-ce qu'elles prennent en compte la façon dont les interactions verbales authentiques se déroulent? Nous allons justement tenter de répondre à ces questions dans la partie suivante.

La description thématique de la série Plus belle la vie, ses objectifs, la façon dont les producteurs et les scénaristes la conçoivent, son attachement au réel, sa présentation de scènes de la vie quotidienne, sont autant d'arguments qui semblent plaider en la faveur de son utilisation à des fins didactiques. En effet, certaines scènes semblent correspondre, au moins en partie, au manque de ressources mentionné par les enseignants de FLE. L'analyse des dialogues de la série va nous permettre d'avoir un regard sur le réel des interactions verbales des personnages. 


\section{Analyse des interactions de la série et comparaison avec des interactions authentiques}

Nous rappelons que notre question de départ consistait à savoir si nous pouvions préconiser l'usage de la série Plus belle la vie en séance de FLE. Une fois que notre choix fut validé par les thématiques abordées dans la série ainsi que par son souci d'être au plus proche de la réalité des téléspectateurs, nous nous sommes interrogée sur les dialogues de la série. En effet, nous souhaitons examiner si le réalisme revendiqué de la série se reflète dans les pratiques langagières produites par les acteurs. Pour répondre à cette question, nous avons entrepris un travail de comparaison des dialogues de la série avec le déroulement d'interactions verbales authentiques spontanées.

La création de dialogues destinés à être lus par des acteurs ou des comédiens présente la double difficulté pour le rédacteur de poser à l'écrit ce qui doit passer pour de l'oral et de penser les interactions telles qu'elles seraient produites en situation naturelle sans être pensées à l'avance. Comme l'explique Kerbrat-Orecchioni [8] en comparant le dialogue théâtral aux conversations ordinaires, la nature du canal diffère. Etant donné que le français écrit diffère du français oral, il nous paraît intéressant d'analyser la façon dont les scénaristes écrivent des échanges oraux. Elle précise néanmoins: "Le dialogue théâtral peut mimer l'improvisation, il peut produire des effets-de-spontanéité, mais qui seront toujours de l'ordre du «pseudo »; même chose des « ratés » : s'il en survient dans le discours des personnages (sous forme de parole simultanée, d'interruptions, de lapsus, ou autres balbutiements), ils seront immédiatement interprétés comme des ratés délibérés (programmés par l'auteur), donc hautement significatifs (alors que tous les ratés ne le sont pas au même degré dans les conversations ordinaires » (Ibid.). En plus de ces constats, il ne nous parait pas du tout évident qu'un scénariste, aussi bon soit-il, ait une conception exacte de la langue orale et encore moins qu'il s'intéresse aux analyses des interactions verbales réalisées par des sociolinguistes.

Nous avons donc entrepris un travail minutieux d'analyse des interactions verbales produites dans la série Plus belle la vie. En nous référant à des analyses interactionnelles, nous avons choisi d'étudier différents phénomènes propres au français parlé en interaction. Nous avons ainsi constitué une liste de phénomènes spécifiques à l'oral et observé leur présence ou leur absence dans les interactions de la série. Le travail de Quaglio [1] présente une étude un peu similaire, mais de plus grande envergure, avec la comparaison de dialogues en anglais de la série Friends et de conversations extraites du Longman Grammar Corpus. L'auteur adopte une approche grammaticale et utilise des traits linguistiques identifiés par Biber [9] comme représentatifs de 
certains aspects de la conversation naturelle comme points de comparaison entre les deux corpus. Dans le cadre de notre étude, nous avons sélectionné un certain nombre de phénomènes langagiers spécifiques au français parlé en interaction. Nous avons comparé leur présence ou leur absence dans les dialogues de Plus belle la vie et dans les interactions du corpus TCOF (http://www.cnrtl.fr/corpus/tcof/).

Deux corpus sont donc utilisés dans le cadre de cette étude : un corpus constitué d'épisodes de la série Plus belle la vie et le corpus TCOF qui est, en quelque sorte, notre corpus de référence. Le premier corpus est constitué de trente épisodes de la série. Ces épisodes ont été diffusés entre 2015 et 2017. Nous avons choisis dix épisodes, qui se suivent, par année. En procédant de cette façon, nous avons pu suivre les intrigues en cours sans trop de difficultés. Il nous a fallu néanmoins, lire les résumés des épisodes précédents et trouver des informateurs-téléspectateurs pour nous situer les personnages et les événements. En outre, de nombreux sites Internet sont consacrés à la série et nous ont permis de trouver les informations nécessaires à la compréhension des épisodes que nous avions choisis. Tous les épisodes ont été transcrits et alignés texte-son avec Transcriber en respectant les conventions de TCOF. Nous remercions les étudiants de Master, déjà mentionnés précédemment, qui ont également participé à cette étude entre 2015 et 2017.

Pour réaliser cette étude, nous avons adopté une démarche qui associe une approche empirique avec les résultats d'études antérieures qui ont repéré des phénomènes spécifiques au français parlé en interaction [notamment 1015]. Une démarche similaire a été adoptée dans le cadre de l'analyse de séquences émotionnées en interaction [16]. Pour évaluer la proximité ou la distance des dialogues de la série avec de réelles interactions, nous avons choisis les phénomènes langagiers et interactionnels suivants: les mécanismes d'alternance des tours de paroles (chevauchements, interruptions), les mots de l'oral, les constructions syntaxiques, les énonciations conjointes, les reprises, les marques d'accord, les hésitations et les piétinements. Chacun des ces phénomènes a déjà été analysé dans des interactions verbales de différentes natures. Par exemple, dans l'extrait suivant, provenant du corpus TCOF, nous pouvons observer leurs actualisations :

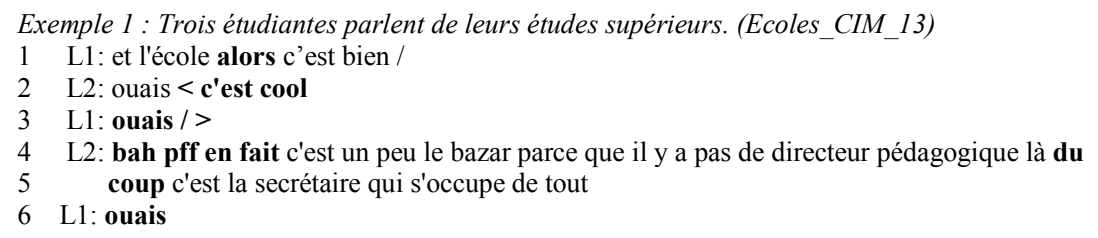


14 L2: et on a des partiels euh au mois de janvier on a genre neurosciences on a jamais eu 15 neurosciences

16 L1: c'est vrai /

17 L2: du coup on sait pas comment ça va se passer c'est un peu le $<$ bazar

18 L1: mais ils > vont peut-être pas vous le mettre là du coup le partiel /

19 L2: je sais pas mais normalement ils sont obligés parce que c'est euh c'est vu que c'est en

20 licence ça doit êt- être pareil dans toute la France + enfin donc c'est un peu le bazar mais 22 euh sinon c'est cool

23 L1: et $<$ vous avez

24 L2: ***>

25 L1: genre euh cours euh tous les jours euh

26 L2: ouais $<$ mais $* * *$ ils sont euh

27 L1: dix heures de cours ou $>$

28 L2: non

29 L1: non

Dans cet extrait, nous avons mis en gras différents éléments qui seront des points de comparaison avec les dialogues de la série. Nous pouvons remarquer une accumulation de certains des phénomènes que nous avons retenus. Les locuteurs se coupent la parole et parlent en même temps (les chevauchement sont signalés par les chevrons $<\ldots>$ ). Certains énoncés sont d'ailleurs inaudibles (signalés par ***, 1.24 et 26 ) parce qu'ils sont produits simultanément. Les locuteurs utilisent de nombreux mots de l'oral pour ponctuer leur discours : alors, bah, en fait, du coup, genre. Ces ponctuants sont très fréquents dans les interactions verbales spontanées. Nous observons des traces de l'élaboration du discours avec des hésitations (euh) et des amorces de mot $(f-, \hat{e} t-)$. L'extrait comporte des marques d'accord ou de confirmation exprimées avec ouais (1.6 par exemple). Nous constatons aussi que L1 en ligne 3 produit une répétition pour poser une question : le ouais avec une intonation montante (marquée par/) incite L2 à poursuivre avec une explication. Nous pouvons également identifier des constructions syntaxiques spécifiques. Par exemple, ligne 14, L2 produit l'énoncé et on a des partiels euh au mois de janvier on a genre neurosciences on a jamais eu neurosciences. Cet énoncé est construit avec des détachements ou une succession de trois unités de construction de tour [ou Turn-Constructional Units, 17]. Enfin, ligne 27, nous constatons une pratique langagière particulière qui permet de poser une question. Bien que l'énoncé dix heures de cours ou soit produit en même temps qu'un autre énoncé, nous pouvons remarquer qu'il s'agit d'un énoncé inachevé qui vise à poser une question. 
Ce procédé a déjà identifié par ailleurs [18]. L'activité langagière visée est réalisée. Il est fort probable que L1 n'ait pas eu l'intention de poursuivre son énoncé, l'alternative proposée suivie de ou suffit pour que L2 comprenne qu'il s'agisse d'une question, d'ailleurs elle répond avec non (1.28). Cette réponse est ensuite répétée par L1 (1.29). Par ailleurs, les reprises sont fréquentes, elles ont différentes fonctions dans les interactions [19], nous pouvons en relever quelques unes dans cet extrait (par exemple : 1.4 c'est un peu le bazar, 1.7 c'est trop le bazar, 1.20 c'est un peu le bazar).

Nous analysons un second exemple du corpus TCOF afin d'illustrer le phénomène d'énonciation conjointe [15] :

Exemple 2 : Extrait d'une discussion entre deux amies qui ont une double nationalité - française et marocaine. Elles discutent des différences culturelles. (Nat_HOU_07)

L1: c'est vraiment ce qu'elle a ce qu'elle m'a transmis c'est

L2: les valeurs

L1: ouais les valeurs qu'elle m'a transmis v- via euh

L2: alors quelles valeurs justement /

Dans cet extrait, L2 complète l'énoncé de son interlocuteur. Elle produit une complétion (les valeurs) du tour de parole de L1. Cette complétion s'inscrit dans la construction syntaxique du tour précédent. L1 ratifie ensuite la complétion avec une marque d'accord et une reprise (ouais les valeurs) et poursuit son discours. Les énonciations conjointes sont des traces de la construction collaborative $\mathrm{du}$ discours. Elles mettent en lumière la coopération des interlocuteurs et montrent de quelle façon ces derniers s'appuient les uns sur les autres pour interagir. Les interactions verbales sont toujours co-construites par les participants. Cette co-construction se reflète dans les pratiques langagières des locuteurs. Nous pouvons également remarquer, dans cet exemple, une trace de l'élaboration du discours de L1 avec un retour en arrière sur l'axe syntagmatique ou une autocorrection. Nous pouvons disposer l'énoncé en grille [10] :

c'est vraiment ce qu'elle a ce qu'elle m'a transmis

La disposition en grille montre les étapes de la confection du discours. Ce court extrait présente également des hésitations ( $v$ - et euh) ainsi qu'une prise de tour de parole à la suite d'une hésitation.

Ainsi, ce que nous pouvons constater c'est que les phénomènes langagiers que nous avons sélectionnés pour analyser le degré de réel des dialogues de la série Plus belle la vie n'apparaissent pas de façon isolée. Ils sont nombreux et fréquents dans des interactions spontanées. Ils sont présents dans quasiment chaque tour de parole des locuteurs. Nous pouvons maintenant analyser les dialogues de la série en prenant comme point de comparaison la présence ou l'absence et la fréquence de ces phénomènes. 
Pour effectuer cette comparaison, nous avons choisi de présenter ici l'analyse d'une séquence complète d'un épisode. En effet, chaque épisode se découpe en plusieurs parties que nous avons appelé des séquences. Chaque séquence est thématique, elle met en scène plusieurs personnages qui échangent à propos de l'une des intrigues de l'épisode. L'extrait que nous présentons est la deuxième séquence de l'épisode 3188 .

Extrait épisode 3188 : Une discussion entre Barbara et Léo, son père, à propos d'Abdel, qui est l'ancien petit-ami de Barbara et qui fait partie de la mafia. La scène se déroule dans la cuisine du bar où travaille Barbara.

1. Léo: tu es toujours amoureuse d'Abdel ou quoi

2. Barbara: bah non non mais tu vois ça m'a fait quelque chose quoi quand on était ensemble

3. bah c'est avec moi + qu'il avait envie de devenir papa

4. Léo: ben enfin depuis qu'il est avec la fille Paolini tu pouvais t'y attendre non

5. Barbara: oui $[\mathbf{p f l}$ non d'ailleurs $\mathbf{j}$ - je sais pas je crois que j'y pensais même pas en fait

6. Léo: oui bah là va falloir que tu t'y fasses parce que dans quelques mois ils vont se balader

7. dans le quartier avec le marmot

8. Barbara: ah bah ça ça risque pas ils s'en vont

9. Léo: comment ça ils s'en vont

10. Barbara: ben ils quittent Marseille

11. Léo: ah bon + pour aller où

12. Barbara: j'en sais rien il m'a pas dit non mais du coup tu vois euh le départ plus le bébé ça

13. + ça me fait bizarre quoi c'est comme si lui il avançait et pas moi

14. Léo: bah bien sûr que si tu as avancé qu'est-ce qu'est-ce que tu racontes

15. Barbara: /// ben après je trouve ça bien hein enfin je veux dire je pense que c'est une bonne

16. chose euh qu'il ait envie de repartir de zéro

17. Léo: ah bon il veut repartir à zéro c'est ce qu'il t'a dit

18. Barbara: ouais + ouais puis je connais Abdel tu vois là il va devenir papa + il va avoir envie

19. d'arrêter les trucs pas clean

20. Léo: ouais + enfin quand on est mêlé à ce milieu de près ou de loin on (n')arrête pas du jour

21. au lendemain hein c'est moi qui te le dis

22. Barbara: ben pourtant il a l'air bien décidé hein

23. Léo: oui bah regarde son père + combien de fois il a voulu arrêter + chaque fois il replonge

La première chose que nous pouvons constater, c'est qu'il n'y a aucun chevauchement de paroles. Ce constat a également été fait par Quaglio [1] lors de son analyse des dialogues de Friends. Le média explique parfaitement cette absence. Les producteurs ne prendraient pas le risque que les paroles prononcées par leurs acteurs ne soient pas audibles. Ainsi, les prises de paroles se font souvent avec des enchainements immédiats. Néanmoins, nous avons rencontré des situations où les personnages parlent en même temps, notamment lorsqu'ils sont énervés. C'est le cas dans l'extrait suivant :

Extrait Episode 2920 : Roland est le propriétaire du bar du Mistral dans lequel Barbara et son ami Francesco sont cuisiniers. Roland pense que ces derniers veulent partir parce qu'ils ont reçu une proposition d'embauche dans un grand restaurant. Il y a un malentendu, les esprits s'échauffent. 
Barbara: mais non mais non mais mais non mais c'est pas vrai écoutez Roland je vais tout vous expliquer

Roland: bon < écoute

Barbara: en fait $>$

Roland: je vais vous dire une bonne chose hein je vous ai fait une proposition je la referai pas si vous êtes trop gourmands vous pouvez toujours aller voir ailleurs

Barbara: alors je < voulais juste

Francesco: c'est toi qui nous l'as > envoyé Frémont hein je viens de me faire engueuler par Chaumette qu'est-ce qu'il t'a pris

Barbara: vous me dites hein quand je peux en placer une

Roland: bon alors écoutez expliquez vous entre vous parce que moi j'en ai ma claque

Les scénaristes choisissent précieusement les moments où les locuteurs parlent en même temps. Cela arrive très rarement et uniquement dans les cas où les personnages s'emportent. D'ailleurs, dans l'extrait ci-dessus, Barbara fait un métacommentaire sur la situation avec: vous me dites hein quand je peux en placer une. Au-delà de ces effets volontaires, déjà relevés par Kerbrat-Orecchioni [8] dans le dialogue théâtral, les chevauchements de parole sont extrêmement rares.

Dans l'extrait de l'épisode 3188 ci-dessus, nous pouvons observer un bon nombre des phénomènes que nous avons identifiés comme étant des caractéristiques du français parlé en interaction. Tout d'abord, la séquence comporte de nombreux mots de l'oral qui permettent aux personnages de ponctuer leur discours : ou quoi, ben non, tu vois, oui non, ah non, ah bah, ben, bah, du coup, hein, enfin. Ces petits mots de l'oral [20], que nous pouvons également appeler marqueurs de discours [21], ponctuants [22], particules énonciatives [23] ou pragmatiques [24], ont des rôles interactionnels et des valeurs pragmatiques spécifiques. Dans la séquence étudiée, ils semblent remplir des fonctions, identifiées par ailleurs, d'insistance (1.1 ou quoi), d'ouverture de tour (1.2 bah, 1.4 ben, 1.8 ah bah, 1.14 bah, 1.22 ben), de fin d'unité de tour (1.2 et 1.13 quoi), d'articulation du discours (1.12 non mais du coup), de surprise (1.4 enfin et non en fin de tour), de demande d'attention $(1.2,1.12,1.18$ tu vois $)$, de marque d'attention ou d'empathie (1.6 oui), de rappel de connivence (1.22 hein) ou encore de reformulation (1.15 enfin je veux dire). Nous pouvons également souligner la présence de oui non ligne 5 . Cette pratique langagière opère un double mouvement : le oui répond à la question posée dans le tour précédent et le non apporte une nuance et annonce un argument. Le couple oui non est fréquemment utilisé à l'oral mais a un fonctionnement tout à fait particulier influencé par la situation de communication [25]. Ainsi, ces marqueurs contribuent à rendre cette interaction proche d'une interaction authentique. La situation de communication, qui met en scène un père et sa fille qui abordent un sujet délicat, légitime la quantité de ces marqueurs dans l'interaction. Réciproquement, ces marqueurs sont suffisamment nombreux, 
associés à d'autres phénomènes langagiers, pour rendre compte de la situation de communication.

Ensuite, les énoncés comportent des traces d'hésitation qui marquent généralement l'élaboration du discours (une amorce de mot $1.5 j$ - et deux euh 1.12 et 1.16). Ce dialogue contient également des répétitions (1.2 non non, 1.18 ouais + ouais $)$. Ces dernières semblent être utilisées pour remplir deux objectifs différents, la première pour insister sur la réponse négative du locuteur et la seconde pour faire avancer le discours. Enfin, cette séquence présente des constructions syntaxiques particulières, propres à l'oral, avec des détachements ou des accumulations d'unités de construction de tour. Nous pouvons mentionner, ligne 2, l'énoncé complexe construit avec une clivée : ça m'a fait quelque chose quoi quand on était ensemble bah c'est avec moi + qu'il avait envie de devenir papa. Avec cet exemple, nous constatons que les énoncés ne suivent pas le modèle syntaxique traditionnel ou canonique: sujet - verbe - complément. Nous pouvons analyser également le dernier énoncé de la séquence, ligne 23 : oui bah regarde son père + combien de fois il a voulu arrêter + chaque fois il replonge. Cet énoncé est composé d'une suite d'unités de construction de tours sans lien entre elles. Nous n'aborderons pas la prosodie, dans cette étude, mais elle a une grande importance dans le réalisme des dialogues. De même, nous avons choisi ici de ne pas mentionner les gestes, les postures et les regards mais ces éléments contribuent également au réalisme de la situation de communication. Ces éléments sont assez bien maitrisés par les acteurs et sont souvent accentués par les plans ou les façons de filmer.

Nous n'analyserons pas plus finement cette séquence, nous avons identifié une accumulation des phénomènes langagiers que nous avions retenus comme étant spécifiques au discours en interaction. A l'issue de notre analyse, nous pouvons reconnaitre que cette accumulation contribue au réalisme de cette interaction. Cependant, toutes les séquences des épisodes de Plus belle la vie ne présentent les mêmes caractéristiques. Certains dialogues sont éloignés des interactions verbales authentiques. Par exemple, dans l'extrait suivant, les dialogues contiennent peu de marques spécifiques aux interactions verbales :

Extrait 1 de l'épisode 3186 : Discussion entre Vanessa et Catherine au domicile de Vanessa qui héberge Catherine. Catherine est la mère du compagnon de Vanessa. Vanessa est la fille d'un chef de la mafia.

Vanessa: vous avez fait connaissance avec Doumé

Catherine: oui on s'est dit bonjour toute à l'heure c'est votre cousin c'est ça

Vanessa: oui il va habiter ici quelques temps

Catherine: vous avez l'air très proches

Vanessa: c'est c'est un peu comme mon petit frère il a grandi avec moi c'est pour ça

Catherine: ah oui 
Vanessa: bah oui son son père et le mien avaient des affaires ensemble quand il y a eu des embrouilles avec les flics c'est son père qui a tout pris sur lui pour protéger la famille

Catherine: et il est resté combien de temps en prison

Vanessa: il est mort là-bas

Catherine: comment ça

Vanessa: bah une bagarre qui a mal tourné et c'est à ce moment là que mon père a recueilli Doumé à la maison et il avait treize ans

Dans cet extrait, nous observons quelques phénomènes langagiers propres au discours oraux. Il y a quelques répétitions (c'est c'est, son son), quelques mots de l'oral (bah oui, bah) et quelques constructions syntaxiques non canoniques. Néanmoins, cet extrait est très joué (sur le plan intonatif) par les actrices et paraît peu naturel. Nous pouvons observer un autre exemple, extrait du même épisode :

Extrait 2 de l'épisode 3186 : Abdel est avocat mais aussi membre de la mafia. Vincent est chef d'une entreprise de BTP qui s'appelle Phénicie. Abdel a assuré la protection du chantier et demande un service en échange. L'interaction se déroule dans un bureau.

Abdel: ça à l'air de bien se passer sur le chantier \

Vincent: oui bon les les les ouvriers ont été un peu surpris par les méthodes de vos vigiles mais euh bon ils ont le mérite d'être intimidants

Abdel: je pense que j'ai trouvé le service que vous pourriez me rendre en échange de cette protection

Vincent: je vous écoute

Abdel: et bien de bon clients à moi souhaiteraient investir dans le BTP

Vincent: et donc

Abdel: et donc je me disais que ce serait intéressant pour eux de rentrer dans le capital de Phénicie à hauteur de vingt pourcent

Vincent: vingt pourcent

Abdel: oui

Vincent: c'est énorme pourquoi ils veulent faire ça

Abdel: pour investir tout simplement ben il se trouve euh qu'ils disposent d'une belle somme en cash

Cet extrait comprend des éléments du français parlé en interaction. Par exemple, le premier énoncé, l'assertion ça à l'air de bien se passer sur le chantier prononcé avec une intonation descendante, présente une façon de poser une question à l'oral. Ce procédé n'est jamais décrit dans les grammaires traditionnelles ou les manuels de langue. La réponse oui produite par Vincent est suivie d'un mot de l'oral bon puis de trois répétitions de les puis d'une hésitation euh. Néanmoins, la suite de l'échange présente peu de phénomènes similaires et le ton des locuteurs est assez peu naturel, ce qui rend l'échange assez éloigné d'une interaction authentique.

Ainsi, en comparant les dialogues de Plus belle la vie avec des interactions verbales authentiques, nous pouvons constater que certains dialogues de la série se rapprochent du français parlé en interaction et que 
d'autres s'en rapprochent un peu moins. Nous avons remarqué que pour qu'un échange paraisse naturel, il ne suffit pas d'intégrer, dans les paroles des acteurs, un ou deux phénomènes propres à l'oral. Les interactions paraissent naturelles lorsque plusieurs phénomènes sont combinés, comme c'est le cas dans les interactions authentiques. L'analyse thématique et interactionnelle des dialogues montre que certaines séquences pourraient être utilisées à des fins didactiques. Néanmoins, il semble qu'une sélection doive être opérée notamment en fonction des objectifs d'apprentissage des apprenants de FLE.

\section{Sélection du corpus Plus belle la vie et expérimentation en cours de FLE}

La sélection des séquences qui composent le corpus Plus belle la vie exploitable à des fins didactiques s'est révélée complexe. Comme nous l'avons vu dans l'analyse des extraits de la série, les dialogues sont assez proches du français parlé en interaction. Même ceux qui en sont éloignés présentent tout de même des phénomènes qui pourraient être intéressants pour des apprenants de FLE. Néanmoins, après que chaque séquence a fait l'objet d'une analyse, nous avons quand même sélectionné celles qui se sont révélées les plus proches des interactions verbales authentiques spontanées et nous avons écarté les séquences les plus éloignées. Cette première sélection est liée à l'objectif de cette étude. Nous avons souhaité trouver des séquences, extraites d'une série télévisée, qui permettent d'exposer les apprenants à la langue cible. Cette exposition doit donc se rapprocher au maximum de la langue réelle, celle que les enseignants de langue ont des difficultés à faire entrer dans leurs cours de FLE. De plus, avec les trente épisodes de notre corpus, nous avions près de 500 séquences. Nous avions donc, d'un point de vue quantitatif, largement de quoi faire du tri. Enfin, il existe près de 4000 épisodes de la série. Si cette étude devait prendre une plus grande ampleur, la sélection ne pose aucun problème. Il restera toujours suffisamment de séquences appropriées utilisables en cours de FLE.

Nous avons également écarté les séquences qui nous ont paru contenir trop de phénomènes spécifiques. En effet, certaines séquences, notamment celles qui mettent en scène des adolescents, présentent une profusion d'expressions à la mode, de petits mots de l'oral parfois mal placés et un lexique particulier qui semble être catégorisé comme la façon de parler des jeunes. Nous avons identifié ce même problème lors d'échanges qui mettaient en scène des personnages de la mafia. A ce propos, nous pouvons 
mentionner qu'une enseignante de FLE a jugé préférable ne pas utiliser les dialogues qu'elle a jugé trop vulgaires. D'autres ont justement jugés que ces mêmes dialogues pouvaient être intéressants justement en raison du lexique rarement enseigné aux apprenants de français mais qui fait partie du lexique presque quotidien. Ainsi, certains échanges semblent intéressants dans la mesure où les scénaristes ont pris en compte les éléments de la situation de communication et font varier les discours produits par les locuteurs. Cependant, certains échanges sont en quelque sorte des "caricatures » d'interactions verbales, ils présentent de nombreux stéréotypes sur des variétés langagières.

Nous avons aussi écarté certaines séquences bien qu'elles présentaient un phénomène interactionnel intéressant. Par exemple, certains échanges auraient pu être utilisés pour illustrer des phénomènes particuliers comme l'énonciation conjointe. Ce phénomène est quasiment inexistant dans les dialogues de Plus belle la vie alors qu'il est fréquent dans les interactions authentiques, quel que soit le genre de discours (conversation, entretien, réunion, etc.). Dans de rares épisodes nous trouvons une sorte d'énonciation conjointe, comme c'est le cas dans les deux extraits suivants :

Extrait de l'épisode 3191 : Alison et Doumé boivent un verre sur la terrasse du bar du Mistral. Alison: bon on trinque à quoi +

Doumé: à la liberté +

Alison: et au quartier

Extrait de l'épisode 3186 : Abdel et Vanessa envisagent de quitter Marseille et le "milieu», c'est-à-dire le milieu de la mafia.

Abdel: on va le quitter le milieu alors on s'en fout de toutes ces conneries

Vanessa: oh ouais ce que je souhaite le plus au monde puis il y aura avec toi Jordan le bébé

Abdel: et mes parents

Ces extraits présentent des complétions (en gras) qui complètent le tour précédent pour constituer une paire que nous avons appelée une énonciation conjointe. Cependant, ces complétions représentent un type de complétion, ce sont des ajouts, et apparaissent dans des séquences peu représentatives du français parlé en interaction (nous ne présenterons pas les analyses ici). Elles ont donc été écartées du corpus.

Ensuite, nous avons écartés des séquences qui sont proches des interactions authentiques mais qui présentent des intrigues invraisemblables. Comme nous l'avons évoqué précédemment, la série met en jeu des scènes de la vie quotidienne mais également des intrigues policières et parfois fantastiques. Ces dernières tiennent en haleine les téléspectateurs et assurent leur fidélité à la série. Cependant, notre objectif étant d'identifier des séquences qui pourraient remplacées des interactions authentiques 
quotidiennes, nous n'avons pas conservé les séquences évoquant par exemple : l'apparition d'un fantôme, les crimes meurtriers de la mafia ou encore les actions d'un tueur en série.

Ainsi, le corpus Plus belle la vie, constitué à des fins didactiques, est composé des deux tiers des séquences des trente épisodes du corpus de travail de départ, soit environ 300 séquences. La sélection a été effectuée selon les critères précédemment explicités. Ce nouveau corpus est transcrit, aligné texte-son, et peut donc être utilisé comme un corpus d'interactions authentiques à des fins didactiques [26-30]. Nous y reviendrons.

Enfin, nous allons présenter une des expérimentations, réalisée en décembre 2017 à partir de ce corpus, avec des étudiantes du Kazakhstan qui ont suivi des cours de FLE au Département de Français Langue Etrangère (DéFLE) de l'Université de Lorraine, à Nancy. Ces étudiantes suivent des cours spécifiques, en cette période les cours traditionnels ont laissé la place aux examens. Notre public est composé de sept jeunes filles kazakhes qui sont arrivées en France trois semaines avant notre expérimentation. Leur séjour en France n'était pas prévu, des cours spécifiques ont donc été mis en place et assurés en partie avec Florence Poncet avec qui nous collaborons pour cette expérimentation. Les étudiantes ont toutes autour de 20 ans et font des études de langues étrangères appliquées dans leur pays. Deux d'entre elles ont un niveau A2 en français, trois un niveau B1, une un niveau B2 et une un niveau $\mathrm{C} 1$. En fonction de ces apprenantes et de leurs objectifs d'apprentissage, nous avons repensé le corpus et choisi des séquences particulières. Nous avons sélectionné des thèmes qui pouvaient les intéresser ou des situations de communication qu'elles étaient susceptibles de rencontrer en France et nous avons écarté les séquences dans lesquelles elles ne pourraient s'identifier à aucun des personnages. Nous présentons ici une partie du travail que nous avons effectué avec une seule séquence de manière approfondie, la séquence que nous avons analysée précédemment extraite de l'épisode 3188 mettant en scène un père et sa fille. Nous avons tout d'abord évalué la compréhension globale de la séquence après une première diffusion visuelle, sans la transcription. Toutes les étudiantes, quel que soit leur niveau de français, ont trouvé l'exercice difficile voire très difficile. Le principal obstacle à la compréhension identifié par les étudiantes est que les personnages parlent très vite alors que nous pouvons qualifier le débit de normal, ni trop lent, ni trop rapide. De façon globale, dans un premier temps, les étudiantes comprennent que les personnages évoquent une troisième personne, la ville de Marseille, un bébé et une relation amoureuse. Dans un deuxième temps, nous avons effectué un travail de compréhension détaillée et les étudiantes finissent par comprendre de plus en plus d'éléments. Nous ne détaillerons pas ce travail mais nous pouvons mentionner les réactions de 
ces étudiantes face à ce document. Elles soulignent que ce document ne peut pas être un reportage ou un documentaire parce que les locuteurs ne parlent pas d'une façon très compréhensible. Elles remarquent que ce qu'elles ont entendu ne correspond pas au français qu'elles ont appris et que leurs enseignants de français ne s'expriment pas de cette façon. Au Kazakhstan, lorsque ces étudiantes travaillent à partir de documents visuels, elles le font avec des documents de TV5 Monde donc des reportages, des films tournés pour le FLE ou des chansons. Elles estiment que l'extrait de la série présente un français familier, notamment parce que tout n'est pas prononcé et que certains passages ne sont pas dans un français « correct ». Après ces premiers travaux et ces premiers échanges, nous avons proposé un court exercice de transcription aux étudiantes (lignes 1 à 5). Lors de cet exercice, nous avons pu constater que tous les éléments que nous avons identifiés comme relevant du français parlé en interaction engendraient des problèmes de segmentation à l'écrit. Par exemple, les segments tu vois ça, c'est avec moi, ensemble bah, enfin depuis, en fait, n'ont pas été correctement transposés à l'écrit. Cet exercice a surtout été un prétexte pour faire remarquer aux étudiantes les spécificités d'une interaction verbale. Chaque spécificité a fait l'objet d'un travail de compréhension particulier, à l'aide de la transcription. En conclusion de cette expérimentation, nous pouvons évoquer que nos étudiantes étaient ravies d'être confrontées à du français parlé en interaction (même si nous leur avons expliqué qu'il s'agissait d'une série) notamment parce qu'elles ont relevé dans cet extrait des éléments qu'elles avaient déjà entendu en arrivant en France mais qu'elles ne comprenaient pas, c'est le cas de $d u$ coup, par exemple. Elles ont également apprécié d'être confrontées à une autre variété de langue, à laquelle elles n'avaient jamais eu accès jusqu'à présent. Elles nous ont demandé des renseignements sur la chaine et l'horaire de diffusion de la série, pour continuer à entrainer leur compréhension. Enfin, elles ont noté les expressions qui les ont marqué, comme tu vois, et se sont données comme défi de les utiliser de façon appropriée dans leurs prochaines situations de communication, notamment en fonction de leurs interlocuteurs. En bref, nous constatons que l'utilisation de séquences de la série Plus belle la vie soulève le même enthousiasme que l'utilisation de corpus multimédia de situations de communication authentiques mais la série a le mérite de proposer presque 4000 épisodes.

D'autres expérimentations montrent que l'observation avec un concordancier de l'apparition de certains traits langagiers, tels que l'utilisation des blocs de mots ou des chunk je pense que, tu vois ou je veux dire est possible avec ce corpus. En effet, notre corpus Plus belle la vie est transcrit et aligné texte-son avec Transcriber. Nous utilisons le concordancier Jconc (aligné texte-son) pour rechercher avec les apprenants 
les phénomènes langagiers de leur choix et analyser leurs apparitions et leur sens en contexte. Nous pouvons imaginer que ce corpus puisse également être étiqueté, notamment d'un point de vue pragmatique pour que les apprenants puissent observer quelles pratiques langagières accomplissent quelles activités langagières dans des genres de discours et des situations de communication particuliers. L'observation et l'analyse des données constituent les premières phases du processus d'acquisition de la langue.

\section{Conclusion}

L'objectif de cette étude était de trouver des vidéos qui pourraient pallier le manque de données authentiques utilisables en cours de FLE. Nous avons choisi de travailler avec des épisodes de la série Plus belle la vie, notamment parce que cette série est réaliste et qu'elle met en scène des interactions de la vie quotidienne des Français. L'analyse des dialogues montre que certaines séquences de la série sont proches des interactions authentiques, analysées par ailleurs. Nous avons donc constitué un corpus de séquences des épisodes de la série qui nous ont paru pertinentes à utiliser en cours de FLE. Les premières expérimentations révèlent l'utilité d'une telle démarche. Les apprenants ainsi que les enseignants de FLE ont montré un grand intérêt pour cette démarche.

Les principes qui sous-tendent cette étude et cette méthodologie didactique sont ceux de l'apprentissage sur corpus et de l'utilisation des corpus à des fins didactiques. Il existe plusieurs façons d'entrer dans des corpus pour des apprenants de langue. La première est d'utiliser chacune des ressources comme des documents authentiques. L'observation, par les apprenants, du déroulement des interactions, des activités langagières mises en œuvre et des pratiques langagières contextualisées, contribue à un apprentissage de la langue sur objectifs spécifiques et individuels. La seconde est l'interrogation des données avec des outils de la linguistique de corpus, principalement avec un concordancier. L'observation des occurrences et l'analyse de leurs apparitions en contexte facilitent également l'apprentissage et l'acquisition de la langue cible. L'utilisation d'un corpus tel que celui de Plus belle la vie n'est pas différente. Cependant, ce corpus permet de présenter aux apprenants une grande variété de situations de communication.

\section{Références bibliographiques}


1. P. Quaglio, Television dialogue: The sitcom Friends vs. natural conversation (John Benjamins Publishing, 2009).

2. P. Forchini, Movie language revisited. Evidence from multi-dimensional analysis and corpora (Peter Lang, 2012).

3. M. Boutet, Histoire des séries télévisées. In S. Sepulchre (Eds). Décoder les séries télévisées (Bruxelles, De Boeck, p.11-46, 2011)

4. J. Y. Le Naour, Plus belle la vie. La boîte à histoires (Presses universitaires de France, 2013).

5. M. Mille. Rendre l'incroyable quotidien. Fabrication de la vraisemblance dans Plus belle la ville. Réseaux, 2011/1.165. p. 53-81. (2011)

6. C. Bryon-Portet, La dimension politique de la série Plus belle la vie. Mixophilie, problématiques citoyennes et débats socioculturels dans une production télévisuelle de service public. Mots. Les langages du politique, 99, 97-112. (2012)

7. C. Ghys, «Plus belle la vie», Mistral gagnant. Libération, samedi 23 avril, p.30-31 (2016).

8. C. Kerbrat-Orecchioni, Dialogue théâtral vs conversations ordinaires, Cahiers de praxématique, 26 (1996)

9. D. Biber, Variation across speech and language (Cambridge: Cambridge, 1988)

10. C. Blanche-Benveniste, Le français parlé (Paris, CNRS Editions, 1990).

11. L. Mondada, Pour une linguistique interactionnelle. In Santacrose M. (Ed.), Faits de langue - Faits de discours. Données, processus et modèles. Qu'est-ce qu'un fait linguistique? Vol 2. (Paris, L'Harmattan, p.95-136. 2002)

12. C. Kerbrat-Orecchioni, Le discours en interaction (Paris, Armand Colin, 2005)

13. V. Traverso, L'analyse des conversations (Paris, Nathan, 1999)

14. V. Traverso, Décrire le français parlé en interaction (Ophrys, Paris, 2016).

15. V. André, L'énonciation conjointe: trace et ressource de la construction collaborative du discours. In SHS Web of Conferences (Vol. 8, pp. 1891-1904). EDP Sciences (2014).

16. M. Quignard, et al. Une méthode instrumentée pour l'analyse multidimensionnelle des tonalités émotionnelles dans l'interaction. In SHS Web of Conferences (Vol. 27, p. 15004). EDP Sciences (2016).

17. E. A. Schegloff, Sequence organization in interaction: Volume 1: A primer in conversation analysis (Cambridge, Cambridge University Press, 2007).

18. J.-M. Debaisieux, A. Boulton, Alors la question c'est...? Questions pragmatiques et annotation pédagogique des corpus. Cahiers de l'AFLS., 13(2), 31-59 (2007).

19. V. André, Eléments de construction collaborative du discours au sein de réunions de travail: la reprise et le couple oui non. Pratiques, $\mathrm{n}^{\circ} \mathbf{1 4 7 / 1 4 8}$, p.199-222 (2010).

20. O. Ducrot et al., Les mots du discours (Paris, Les éditions de minuit, 1980)

21. D. Schiffrin, Discourse Markers (Cambridge, Cambridge University Press, 1987) 
22. D. Vincent, Les ponctuants de la langue et autres mots du discours (Québec, Nuit Blanche Editeur, 1993)

23. J. Fernandez, Les particules énonciatives dans la construction du discours (Paris, Presses Universitaires de France, 1994)

24. K. Beeching, Gender, Politness and Pragmatic Particles in French (Amsterdam, John Benjamins, 2002)

25. V. André, Oui non: une pratique discursive sous influence. Marges linguistiques, 9, p.195-213 (2005).

26. T. Johns, Should you be persuaded : Two examples of data-driven learning. Dans T. Johns, P. King, (éds.), Classroom Concordancing, English Language Research Journal, 4, p.1-16. (1991)

27. G. Aston, Learning with Corpora (Houston, Athelstan, 2001).

28. A. Boulton, H. Tyne, Des documents authentiques aux corpus. Démarches pour l'apprentissage des langues (Paris, Didier, 2014).

29. E. Ravazzolo, V. Traverso, E. Jouin, G. Vignier, Interactions, dialogues, conversations : l'oral en français langue étrangère (Hachette, 2015)

30. V. André, Un corpus multimédia pour apprendre à interagir en situations universitaires en France. Actes du colloque ATPF enseigner le français: s'engager et innover, p.292-316 (2017). 